\title{
INFLUENCE OF COOLED INTERSTELLAR GAS ON THE FUNDAMENTAL PLANE FOR ELLIPTICAL GALAXIES
}

\author{
William G. Mathews ${ }^{1}$ and Fabrizio Brighenti ${ }^{1,2}$ \\ ${ }^{1}$ University of California Observatories/Lick Observatory, Board of Studies in Astronomy and \\ Astrophysics, University of California, Santa Cruz, CA 95064 \\ mathews@lick.ucsc.edu \\ ${ }^{2}$ Dipartimento di Astronomia, Università di Bologna, via Ranzani 1, Bologna 40127, Italy \\ brighenti@bo.astro.it
}

\begin{abstract}
We explore here the possibly important influence of cooled interstellar gas on the fundamental plane of elliptical galaxies. Interstellar cooling is described by a parameterized sink term in the equation of continuity. Parameters that give the best fits to the X-ray observations of elliptical galaxy NGC 4472 are used as a template for other galaxies. These spatially-dependent cooling parameters are then extended homologously to elliptical galaxies of lower mass for which X-ray observations are currently unavailable or impossible to observe because of stronger relative X-ray emission from binary stars. Most of the interstellar gas cools within an effective radius where it can contribute an additional 10 - 30 percent to the mass of the old stellar population. The absence of observed cooled gas and simple theoretical arguments support the hypothesis that the cooled gas is forming into low mass stars, with implications that depend on the IMF of this young stellar population. If the IMF includes only stars of very low mass, $\ll M_{\odot}$ as is commonly believed, the cooled mass is optically dark. For this IMF the mass to light ratios determined from stellar velocities systematically overestimate that of the old stellar population. Furthermore, the total mass and spatial distribution of the optically dark stellar population does not scale homologously with galactic luminosity or radius so the total stellar mass to light ratio is expected to vary with galactic radius. These variations in stellar population and non-homology can introduce pronounced deviations from the fundamental plane. We investigate cooled gas perturbations to the mass to light ratio for several idealized homologous elliptical galaxies and show that they may be incompatible with the observed thinness of the fundamental plane. However, if the IMF of the stellar population produced from cooled interstellar gas is optically luminous, its influence on observed stellar mass to light ratios and the fundamental plane would be lessened. If the IMF of a young, continuously forming and optically luminous stellar population were sufficiently wellbehaved, it could account for about ten percent of the optical light from large elliptical galaxies within $r_{e}$, possibly having important implications for understanding the fundamental plane.
\end{abstract}

Subject headings: galaxies: elliptical and lenticular - galaxies: structure - galaxies: fundamental plane galaxies: cooling flows - x-rays: galaxies

\section{INTRODUCTION}

The mass of hot interstellar gas that cools over a Hubble time in a massive elliptical galaxy is typically $5-7$ percent of the total baryonic mass of these galaxies. Several observations indicate that this gas cools in a significant volume of the in- ner galaxy: (1) the total cooled mass significantly exceeds the mass of central massive black holes (Magorrian et al. 1998), (2) although much of the interstellar gas within $r_{e}$ is ejected from old galactic stars which typically have a systematic galactic rotation, the inner X-ray images of rotating ellipti- 
cal galaxies are nearly circular, implying that the gas cools before it flows very far toward the rotation axis (Hanlan \& Bregman 2000; Brighenti \& Mathews 2000b), (3) direct evidence of diffuse cooled gas at $T \sim 10^{4} \mathrm{~K}$ is invariably observed in large elliptical galaxies in $r \lesssim 0.3 r_{e}$ (e.g. Macchetto et al. 1996), and (4) evolutionary models for the hot interstellar gas are in better agreement with X-ray observations when cooling mass loss is included; most of this gas cools within an effective radius (Brighenti \& Mathews 2000a).

In view of the very small masses of cold $\mathrm{HI}$ or $\mathrm{H}_{2}$ gas that have been observed in elliptical galaxies, far less than the mass of hot gas that has cooled, it is usually assumed that the cooled interstellar gas is continuously forming into a population of very low mass (brown dwarf) stars (Fabian, Nulsen \& Canizares 1982). Such stars add mass but no light. We show here that if the cooled gas is optically dark, the fundamental plane for elliptical galaxies is likely to be perturbed beyond observed limits. Alternatively, if the mass to light ratio of the forming stars is not infinite but is comparable to that of the old stars, then some of the cosmic scatter or tilt of the fundamental plane from the virial plane could be due to the presence of this young stellar population. Support for a continuously forming, optically luminous stellar population in elliptical galaxies is provided by the nearly solar mass of neutral cores in interstellar clouds at which gravitational collapse first occurs, $\sim 2 M_{\odot}$ (Mathews \& Brighenti 1999a), and by the apparent young stellar ages of many elliptical galaxies based on the $\mathrm{H} \beta$ photometric index (Worthey 1994; Worthey, Trager \& Faber 1996; Trager 1997; Mathews \& Brighenti 1999b).

In the following discussion, however, we adopt the usual hypothesis that newly forming stars in elliptical galaxies are optically dark. In this case radial variations in the total stellar mass to light ratio must occur. Furthermore, the total mass of cooled gas varies with galactic luminosity, resulting in rather profound excursions from the fundamental plane, evidently in violation of the remarkable thinness and regularity of this plane.

\section{THE FUNDAMENTAL PLANE}

If elliptical galaxies were perfectly homologous stellar systems with identical stellar popu- lations, then the virial theorem $\sigma_{o}^{2}=\kappa_{v}\left(M / r_{e}\right)=$ $\kappa_{v}\left(L_{V} / r_{e}\right)\left(M / L_{V}\right)$ would imply simple correlations among observable parameters. Here $\sigma_{o}$ is the "central" velocity dispersion, $M$ is the stellar (or galactic) mass, $r_{e}$ is the effective (half-light) radius and $\kappa_{v}$ is a constant of proportionality. The three observables $-\sigma_{o}^{2}, L_{V}$, and $r_{e}$-define a homologous plane (HP) in this (logarithmic) three-dimensional parameter space. Instead, elliptical galaxies satisfy a different, slightly steeper fundamental plane (FP) relation $\sigma_{o}^{2} \propto\left(L_{V} / r_{e}\right)\left[r_{e}^{0.22} \sigma_{o}^{0.49}\right]$, implying that $\kappa_{v}\left(M / L_{V}\right) \propto r_{e}^{0.22} \sigma_{o}^{0.49} \propto M^{0.24} r_{e}^{-0.02} \propto$ $L_{V}{ }^{0.32} r_{e}^{-0.03}$ (Dressler et al. 1987; Djorgovski \& Davis 1987). In view of the importance of elliptical galaxies for $D_{n}-\sigma_{o}$ distance determinations, considerable effort has gone into understanding the physical origins of the "tilt" of the FP relative to the HP.

Interpretations of this tilt are constrained by the regularity of the FP-HP deviation across the entire region observed and the remarkably small observational scatter $(\sim 12 \%)$ about the FP relation (Renzini \& Ciotti 1993). Generally, the FPHP tilt has been interpreted as some combination of two limiting effects: either (1) stellar populations or dark matter contributions vary systematically across the FP so that $M / L_{V} \propto L_{V}^{0.32}$ or (2) homology fails so that $\kappa_{v} \propto L_{V}{ }^{0.32}$. This relation depends mildly on photometric band (e.g. Scodeggio et al. 1998; Pahre \& Djorgovski 1997).

Several types of stellar population variation (age, metallicity or IMF) have been considered along the FP (Renzini \& Ciotti 1993; Pahre, Djorgovski \& Carvalho 1995; Pahre \& Djorgovski 1997). These attempts to interpret the FPHP tilt have not been entirely successful since the $\mathrm{FP}$ is nearly invariant to observed wavelength over the $B-H$ photometric bands. The small systematic wavelength trend of the $\mathrm{FP}$ slope is consistent with the color-magnitude relation for giant elliptical galaxies (Scodeggio et al 1998; Pahre, de Carvalho \& Djorgovski 1998). Moreover, if the stellar population changes across the FP, differential stellar evolution can in some cases introduce a change in the FP slope at large redshifts, but no such effect has yet been observed. It is generally asserted that the full FP tilt cannot be understood solely by metallicity variations or changing stellar populations along the FP since this would require a high degree of fine tuning in the IMF. If $M / L$ is 
assumed to be constant with galactic radius, the mass to light ratios for elliptical galaxies found from dynamic models vary in a manner similar to the FP deviation, $M / L \propto L^{0.35 \pm 0.05}$ (van der Marel 1991).

A wide variety of non-homologous structural and/or kinematic models have been proposed to explain all or part of the FP-HP tilt (e.g., Capelato et al. 1995; Djorgovski 1995; Hjorth \& Madsen 1995; Ciotti, Lanzoni \& Renzini 1996). In these types of models the stellar $M / L_{V}$ does not vary so the FP tilt should be strictly independent of wavelength apart from mutually non-homologous stellar and dark matter distributions. Graham \& Colless (1997) fit the stellar mass and light distribution with Sersic $r^{1 / n}$ laws and note that $n$ increases with galactic mass (by $\sim 2-4$ ) in a non-homologous fashion across the FP. If these galaxies are mapped onto the $\mathrm{FP}$ with an $r^{1 / 4}$ law in the usual way, the resulting $\mathrm{FP}, r_{e} \propto$ $\sigma_{o}^{1.33 \pm 0.10} \Sigma_{e}^{-0.79 \pm 0.11}$, tilts even further from the $\mathrm{HP}, r_{e} \propto \sigma_{o}^{2} \Sigma_{e}^{-1}$, where $\Sigma_{e} \propto L / r_{e}^{2}$ is the surface brightness. However, a dynamical non-homology must accompany a structural non-homology. Solving the Jeans equation for elliptical galaxies modeled with $r^{1 / n}$ profiles (Ciotti 1991; Ciotti \& Lanzoni 1997), Graham \& Colless (1997) and Graham (1998) determine infinite-aperture $\sigma_{t o t, n}$ which when fit to $r^{1 / n}$ galaxies result in $r_{e, n} \propto$ $\sigma_{\text {tot }, n}^{1.72 \pm 0.24} \Sigma_{e}^{-0.74 \pm 0.09}$, which is somewhat closer to the HP. Busarello et al. (1997) consider a different dynamic non-homology based on partial rotational support within $r_{e}$. Using a modified $\tilde{\sigma}$ which incorporates both rotation and random velocities, they find that more than half of the FP-HP tilt is accounted for, $r_{e} \propto \tilde{\sigma}^{1.53 \pm 0.2} \Sigma_{e}^{-0.92 \pm 0.1}$. Busarello et al. claim that the remaining discrepancy can be understood with spatial non-homology and stellar population differences (e.g. Prugniel \& Simien 1997).

Another approach is the possibility that dark (halo) matter makes a variable contribution along the FP (Ciotti, Lanzoni, \& Renzini 1996), resulting in the FP-HP tilt. However, these authors stress again the tightness of observational scatter about the FP so that any systematic trend in the dark matter content must be rather finely tuned. This high degree of coordination may seem unlikely if elliptical galaxies are produced by a variety of random mergers among stochastically differ- ent galaxies of lesser mass. The amount and radial distribution of dark halo matter in the centers of elliptical galaxies is complicated by the baryonic winds in first generation galaxies and the dissipational baryonic concentration expected as giant elliptical galaxies form by mergers; these counteracting effects respectively contract and expand the dark matter distribution relative to the baryonic stellar population.

There is at present no clear winner among the various explanations for the FP-HP tilt. Instead, a patchwork of small contributions - stellar population variations, rotational support and nonhomology - are required to bring FP into agreement with HP (Ciotti, Lanzoni, \& Renzini 1996: Prugniel \& Simien 1997; Pahre, de Carvalho \& Djorgovski 1998). However, there is no compelling reason why these quite different and presumably independent contributions should combine in a coordinated fashion to produce the full FP-HP tilt observed across the entire FP. Moreover, a multiplicity of coordinated explanations for the tilt, none entirely dominant, makes the tightness of the FP even more remarkable.

\section{COOLING DROPOUT MASS AND LOW MASS STARS}

In this paper we introduce a new complication to the FP-HP controversy: a variation in the dynamical $M / L_{B}$ with galactic mass due to the deposition of cooled interstellar gas in $r \lesssim r_{e}$. The importance of cooled gas in the galactic mass budget follows from the universality of hot interstellar gas in giant elliptical galaxies and the direct relationship of the cooled mass to the loss of energy implied by the observed X-ray emission $L_{x}$. For example, interstellar gas in the large Virgo elliptical galaxy NGC 4472 with temperature $T \approx$ $1.3 \times 10^{7} \mathrm{~K}$ emits $L_{x}(0.5-4.5 \mathrm{keV})=4.5 \times 10^{41}$ ergs s${ }^{-1}$ in the Einstein band. The bolometric Xray luminosity, $L_{x, b o l} \approx 1.6 L_{x}(0.5-4.5 \mathrm{keV})$, is the product of the mass cooling rate $\dot{M}$ and the specific enthalpy of the gas, therefore

$$
\dot{M}=\left(\frac{2 \mu m_{p}}{5 k T}\right) L_{x, b o l} \approx 2.5 M_{\odot} \mathrm{yr}^{-1} .
$$

Since $L_{x}$ was somewhat larger in the past, over a Hubble time $\sim 3-4 \times 10^{10} M_{\odot}$ of hot gas will have cooled. Although this amounts to only about 
$\sim 5-10$ percent of the total stellar mass in NGC $4472, M_{* t}=4.73 \times 10^{11} M_{\odot}$, most of the cooled gas is likely to be deposited near the galactic center $r \lesssim r_{e}$ where the hot gas density and X-ray emissivity are greatest and where its contribution to the dynamical $M / L_{B}$ determined from stellar velocity dispersions is most apparent. (In evaluating $M_{* t}$ we assume $M / L_{B}=6$, as explained below.)

Although there is little doubt that interstellar cooling has occurred, two main questions must be addressed: (1) What is the final physical state of the cold gas? and (2) What is the radial distribution of the cooled mass in the galaxy? For many years it has been assumed that low mass stars are the most likely final state for cooled gas (e.g. Fabian, Nulsen \& Canizares 1982). In a recent paper (Mathews \& Brighenti 1999a) we describe how the hot interstellar gas cools (initially) to $T \sim 10^{4} \mathrm{~K}$ where it can be heated and ionized by stellar UV photons and made visible in optical emission lines. This first stage of cooling occurs at many $\left(\sim 10^{6}\right)$ cooling sites distributed over the inner galaxy. However, when the radius of partially cooled gas at $10^{4} \mathrm{~K}$ exceeds the local Stromgren radius at a cooling site, a cold neutral atomic or molecular core forms with $T_{c} \sim 15 \mathrm{~K}$ and $n_{c} \sim 10^{5} \mathrm{~cm}^{-3}$ in pressure equilibrium with the ambient hot interstellar gas. As the mass of the neutral core $M_{c}$ increases further, it becomes Jeans unstable when $M_{c} \sim 1-2 M_{\odot}$ so only stars of this or lower mass can form from the available gas (Ferland, Fabian \& Johnstone 1994; Mathews \& Brighenti 1999a). Cooling gas is processed within HII cooling site clouds with star-forming cold cores in time scales $\left(\sim 10^{5}\right.$ years $)$ that are short compared to their hydrodynamic disruption times in the cooling flow atmosphere (Mathews \& Brighenti 1999a). The high efficiency of low mass star formation nicely accounts for the absence of observable cold $\mathrm{HI}$ or molecular gas in large elliptical galaxies, e.g. for NGC $4472 M_{H I}+M_{H 2}<10^{7}$ $M_{\odot}$. We conclude that two stellar populations coexist in giant elliptical galaxies: an old luminous stellar component with an IMF similar to Salpeter, and a second population of low mass stars continuously forming from the interstellar medium and having a bottom heavy IMF. Although the detailed character of this second IMF is unknown, for the purposes of the following discussion we adopt the usual assumption that it is restricted to very low masses, i.e. its mass to light ratio is essentially infinite.

The second question - concerning the radial distribution of cooled gas mass - can be answered in an approximate way by comparing hydrodynamic models with the radial properties of interstellar gas observed in X-rays. The production of low mass stars at many distributed sites can only occur if the interstellar gas is inhomogeneous. Radiative cooling is locally enhanced in regions of low entropy (low $T$, high density $n$ ). As gas in the galactic cooling flow slowly proceeds inward, regions of progressively higher entropy cool at smaller galactic radii; regions having low entropy cool further from the galactic centers. Distributed $\mathrm{H} \alpha+[\mathrm{NII}]$ line emission observed in the central regions of giant elliptical galaxies illuminate the cooling sites where this process is occurring. Interstellar entropy variations can be created by a variety of complex events: stellar disturbances (e.g. stellar winds or Type Ia supernovae), interactions with merging dwarf galaxies or differential Type II supernova heating in the early universe during the earliest phases of star formation. Because of our poor understanding of these interstellar entropy fluctuations, it is not possible to determine from first principles where most of the hot gas cools and deposits its mass into low mass stars, although the observed optical line emission is a visible tracer of this process. The best currently available means of setting limits on the radial distribution of cooling gas in elliptical galaxies is to compare the observed radial distribution of interstellar X-ray emission with the computed X-ray emission from gas-dynamic models for the cooling flow gas in which cooling dropout is assumed to occur according to various radial prescriptions. We have recently computed a series of models for the large elliptical galaxy NGC 4472 with a variety of assumed mass dropout functions (Brighenti \& Mathews 2000a). In the following section we describe the cooling dropout properties of models that best fit the X-ray observations, then we discuss the implications of this dropout mass for the fundamental plane. 


\section{DESCRIPTION OF HYDRODYNAM- ICAL MODELS}

In view of the uncertainty concerning the cooling dropout, in our gas dynamical models of galactic cooling flows we parameterize the radial mass dropout of cooling gas by introducing an adjustable sink term in the equation of continuity,

$$
\frac{\partial \rho}{\partial t}+\frac{1}{r^{2}} \frac{\partial}{\partial r}\left(r^{2} \rho u\right)=\alpha \rho_{*}-q(r) \frac{\rho}{t_{d o}} .
$$

Here $\rho(r, t)$ and $u(r, t)$ are the gas density and velocity in the cooling flow. The sink term for cooling flow dropout is characterized by a dimensionless parameter $q(r)$ and $t_{d o}=5 m_{p} k T / 2 \mu \rho \Lambda$ is the time for gas at temperature $T(r, t)$ to cool locally by radiative losses at constant pressure (see, e.g. Sarazin \& Ashe 1989). The source term $\alpha(t) \rho_{*}(r)$ represents the rate that interstellar gas is supplied by stellar mass loss from the old stellar population having density $\rho_{*}(r)$. Since $\alpha(t) \propto\left(M / L_{B}\right)^{-1}$ for a wide range of power law IMFs, the total stellar mass ejected $\int \alpha(t) M_{*} d t$ is nearly identical for elliptical galaxies with the same $L_{B}$ but different IMFs (Brighenti \& Mathews 2000a). Following the approach in Brighenti \& Mathews (2000a), we consider galactic cooling flow models for NGC 4472 using a parameterized dropout coefficient

$$
q(r)=q_{o} \exp \left(-r / r_{d o}\right)^{m}
$$

where $q_{o}, r_{d o}$ and $m$ are adjustable parameters. For all sets of dropout parameters considered we found that the total dropout mass in $r \lesssim r_{e} / 3$ is a substantial fraction $(0.10-0.35)$ of the mass of luminous stars in this region. The radial distribution of the dropout mass, assumed to remain at the cooling site, does not follow the space density corresponding to the de Vaucouleurs light profile.

If low mass stars formed from cooling dropout contribute mass but no appreciable light, the hypothesis that we want to test, it follows that the intrinsic "stellar" mass to light ratio of the luminous stellar population in NGC 4472 must be somewhat lower than values determined from the observed motion of luminous stars: $M / L_{B} \approx 9.2$ (van der Marel 1991) or $M / L_{B} \approx 10.3$ (Faber et al. 1997). The most satisfactory mass dropout coefficients $q(r)$ generate hydrodynamic solutions for the interstellar gas at the current time $\left(t_{n}=13\right.$ Gyrs) that reproduce best the observed X-ray image of NGC 4472. Using this approach, we find that the dynamical mass to light ratio in successful hydrodynamic models requires a somewhat lower intrinsic mass to light ratio for the luminous stars $M / L_{B} \approx 6$. For the low mass dropout stellar population we assume $\left(M / L_{B}\right)_{d o}=\infty$.

Ideally, it would be desirable to determine if the same (or appropriately scaled) mass dropout coefficient $q(r)$ used in gas dynamical models for elliptical galaxies of varying $L_{B}$, spread across the fundamental plane, can fit the observed X-ray surface brightness distributions $\Sigma_{x}(R)$ in these same galaxies. Unfortunately, at the present pre-Chandra time sufficiently accurate $\Sigma_{x}(R)$ are known only for several bright elliptical galaxies in Virgo (e.g., Trinchieri, Fabbiano \& Canizares 1986), all having similar $L_{B}$. Approximate $\Sigma_{x}(R)$ distributions are available only for about a dozen elliptical galaxies altogether (Mathews \& Brighenti 1998a). In addition, emission from low mass binary stars begins to dominate the X-ray luminosity in elliptical galaxies several times less luminous than NGC 4472, masking the X-ray emission from the cooling flows. Finally, even though $L_{x} \propto L_{B}^{2}$ approximately holds among brighter elliptical galaxies, there is an enormous spread in values of $L_{x}$ for given $L_{B}$. We believe that most of this spread is due to environmental interactions: (i) tidal exchange of halo hot gas (and dark matter) in the group environments in which these elliptical galaxies formed (Mathews \& Brighenti 1998b) or (ii) ram pressure stripping.

With these difficulties in mind, we chose instead to explore the relative influence of optically dark interstellar dropout not directly on the FP but on a homologous galactic plane (HP) that is scaled from NGC 4472 for which good X-ray observations are available. We consider a sequence of three massive elliptical galaxies, NGC 4472 and two similar model galaxies, $4472 / 4$ and $4472 / 16$, having optical luminosities $L_{B}$ that are respectively 4 and 16 times less than NGC 4472. To explore only the structural non-homology caused by cooling dropout, we also assume that the old stellar population is invariant along our HP, i.e. its $M / L_{B}$ is constant. For perfect homology we also require that the mass and scale radius of the dark halo distribution scale with the baryonic mass. Finally, we solve for the evolution of hot gas in each galaxy along the HP using identical (or scaled) parameters $\left(q_{o}, r_{d o}\right.$ and $\left.m\right)$ that give good agree- 
ment with the X-ray observations for NGC 4472 , $T\left(r, t_{n}\right)$ and $n\left(r, t_{n}\right)$; this procedure is described in more detail in Brighenti \& Mathews (2000a). By this means we can determine the differential, nonhomologous distribution of optically dark dropout mass and assess the deviation of these three idealized galaxies from the HP relation. We expect that these deviations from the HP are similar to deviations of galaxies of similar $L_{B}$ from the local FP. If our procedure were perfectly correct - i.e., elliptical galaxies are exactly homologous and contain identical old stellar populations - we would find that the non-homology introduced by cooling dropout would explain the "tilt" of the observed FP from the HP. Unfortunately, this goal has not yet been achieved.

For perfect homology and simplicity we assume that 4472 and its two clones of lower mass are spherical with de Vaucouleurs stellar profiles and scaled NFW dark halos (Navarro, Frenk, \& White 1996). The effective radius of NGC 4472 is $r_{e}=8.57 \mathrm{kpc}$ (for distance $d=17 \mathrm{Mpc}$ ) and its total (old) stellar mass is $M_{*, t}=4.73 \times 10^{11}$ $M_{\odot}$, based on $M / L_{B}=6$. The NFW halo in NGC 4472 has a virial mass of $M_{h, v i r}=4 \times 10^{13}$ $M_{\odot}$. These parameters give a good fit to the X-ray mass profile of NGC 4472 assuming hydrostatic equilibrium although the NFW profile is found to be somewhat too centrally peaked (Brighenti \& Mathews 2000a). For the smaller comparison galaxies $(4472 / 4$ and $4472 / 16)$ we choose identical stellar parameters but scale the size of the stellar distribution downward by $4^{-0.793}=0.333$ or $16^{-0.793}=0.111$ where the exponent is found from the observed $M_{V}-r_{e}$ relation for massive elliptical galaxies, $r_{e} \propto L_{V}^{0.793} \propto L_{B}^{0.793}$, (Faber et al. 1997). The virial mass of the NFW halos for the smaller galaxies are reduced by 4 or 16 and the scale radius for these halos varies as $r_{s} \propto M_{h, v i r}^{0.457}$, as required by our chosen cosmology for which the NFW concentration varies as $c \propto M_{h, v i r}^{-0.123}$ (Navarro, Frenk, \& White 1996).

As explained in more detail elsewhere (Brighenti \& Mathews 1999a), our gas dynamical models begin with an overdensity in a simple Einstein-de Sitter cosmology $\left(\Omega=1, \Omega_{b}=0.05\right.$, and $H_{o}=50$ $\mathrm{km} \mathrm{s}^{-1} \mathrm{Mpc}^{-1}$ ) toward which baryonic and dark matter are gravitationally attracted. Galactic stars are assumed to form at time $t_{* s}=1 \mathrm{Gyr}$ and, soon afterwards, Type II supernova energy based on a Salpeter IMF (slope: $x=1.35$, mass limits: $m_{\ell}=0.08$ and $m_{u}=100 M_{\odot}$ ) heats the ambient gas within the accretion shock with 80 percent efficiency (see Brighenti \& Mathews 1999a for details). At $t_{*}=2$ Gyrs the de Vaucouleurs stellar profile and potential is constructed, simulating the results of mergers. For this approximate exploration we ignore the possibility that elliptical galaxies of lower luminosity may have formed slightly earlier. In some models we allow the secondary inflow of cosmic gas to continue throughout the calculation. For comparison, in other models we ignore the secondary infall entirely, crudely simulating a tidal truncation and removal of halo material in a relatively dense group of newly formed galaxies, a process more likely in elliptical galaxies of moderate or low mass.

\section{RESULTS}

In Table 1 we list the main results of a variety of hydrodynamic cooling flow models for 4472 , $4472 / 4$ and 4472/16 with various assumed dropout parameters and assumed amounts of accumulated cosmic gas. Of particular interest are the mass to light ratios evaluated at $r_{e} / 3$ and at a fixed distance of $480 \mathrm{pc}$ from the galactic centers. Recall that $M / L_{B}=6$ is assumed for the luminous stars in all three galaxies and that the observed value for NGC 4472 is $~ 9.2$ (van der Marel 1991) or $\sim 10.3$ (Faber et al. 1997). Models in which the (stellar plus dropout) mass to light ratio for NGC 4472 falls in the range $M / L_{B} \approx 8.5-11$ can be regarded as reasonably successful. However, the $M / L_{B}\left(r_{e} / 3\right)$ entries in Table 1 are enhanced by about 15 percent due to a small amount of NFW dark halo material in this region. Since our fits to the X-ray surface brightness of NGC 4472 are improved if the dark halo is less centrally peaked than NFW (also see Kravtsov et al. 1998; Burkert \& Silk 1997; Navarro \& Steinmetz 2000), we believe that its contribution to $M / L_{B}$ in this region should be removed. Therefore, for comparison with $M / L_{B}\left(r_{e} / 3\right)$ values in Table 1 which contain this NFW excess, the range of acceptable models for NGC 4472 should be revised upward to $M / L_{B}\left(r_{e} / 3\right) \approx 9.8-12.6$ (i.e. $\left.\log \left[M / L_{B}\left(r_{e} / 3\right)\right]=0.99-1.10\right)$. Values of $M / L_{B}$ at $480 \mathrm{pc}$ may be more relevant for comparison with $M / L_{B}$ determinations that are often based on stellar velocity dispersions measured within a 
fixed aperture of a few arcseconds about the galactic centers.

In cooling flows without distributed dropout $\left(q_{o}=0\right)$ the flow proceeds entirely to the origin before cooling and the mass to light ratios at 480 pc greatly exceed observed values for 4472 - such models can certainly be rejected. Evolutionary gas dynamic models with uniform $q_{o}=1$ at all radii and with dropout parameters $\left(q_{o}, r_{d o}, m\right)=(4,2$ $\mathrm{kpc}, 1)$ both fit the observed distribution of interstellar gas temperature and density in NGC 4472 reasonably well at the present time $\left(t_{n}=13\right.$ Gyrs $)$ as illustrated in Figure 1. While the agreement in Figure 1 is acceptable, it is by no means perfect. The apparent gas density (enhanced by dropout emission) exceeds observed densities in the central regions ( $r \lesssim 3 \mathrm{kpc}$ ) of NGC 4472, suggesting that some additional non-thermal support is present; NGC 4472 has a weak extended radio source in this region with an appreciable equipartition pressure (Ekers \& Kolanyi 1978). At large galactic radii $(r \gtrsim 10 \mathrm{kpc}$ ) our models in Figure 1 are slightly lower than the azimuthally-averaged values observed in NGC 4472; this density mismatch can be corrected with small changes in the galaxy formation time $t_{*}$ or the efficiency of SNII energy release (Brighenti \& Mathews 1999a). However, our interest here is not to achieve absolute perfection in fitting the observations of NGC 4472, but to make a differential comparison of 4472 with its less massive, homologous clones; 4472/4 and $4472 / 16$. The additional model with stronger but uniform dropout $q=q_{o}=4$, also included in Table 1 , results in apparent gas temperatures at $t_{n}=13$ Gys that are considerably lower than those observed (Brighenti \& Mathews 2000a); this is due to enhanced $\mathrm{X}$-ray emission from cooling regions throughout the cooling flow.

Several interesting details are apparent in Table 1. Since the dropout mass is deposited nonhomologously, the dynamic mass to light ratio varies with galactic radius, i.e. $M\left(r_{e} / 3\right) / L_{B} \neq$ $M(480 \mathrm{pc}) / L_{B}$. Even when the dropout parameters are scaled homologously from 4472 $\left[\left(q_{o}, r_{d o}, m\right)=(4,2 \mathrm{kpc}, 1)\right]$ to $4472 / 4\left[\left(q_{o}, r_{d o}, m\right)\right.$ $=(4,0.667 \mathrm{kpc}, 1)]$, the resulting $M / L_{B}$ are not equal, i.e. dropout does not scale homologously. Some models in Table 1 were performed with no additional secondary infall gas following the time $t_{*}$ of galaxy formation ( $\mathrm{NO}$ in column 5 ). This procedure is justified by the strong correlation of normalized X-ray sizes $r_{e x} / r_{e}$ with $L_{x} / L_{B}$ noted by Mathews \& Brighenti (1998). We explained this correlation with models of tidally truncated galactic dark halos and (mostly secondary infallen) halo gas in smaller galaxies and the transfer of this material to halos of more massive, centrally located elliptical galaxies, a process which is dynamically expected in galaxy groups where elliptical galaxies are formed. The removal of secondary infallen halo gas is therefore more relevant to $4472 / 4$ and $4472 / 16$ than for 4472 (which may have acquired additional halo gas from neighboring galaxies); note that this late arriving cosmic gas also has a larger influence on $M / L_{B}$ for the smaller galaxies.

The non-homologous dropout behavior listed in Table 1 is illustrated in Figure 2 in which the 4472 $\rightarrow 4472 / 4 \rightarrow 4472 / 16$ variation of $M / L_{B}\left(r_{e} / 3\right)$ and $M / L_{B}(480 \mathrm{pc})$ are plotted for each model. The local fundamental plane, $M / L_{B} \propto L_{B}{ }^{\beta}$, shown with dashed lines, has a slope $\beta=0.24$ that is intermediate between values for the B-band FP taken from Jorgensen, Franx, \& Kjaergaard (1996) and Scodeggio et al. (1998). The dashed lines are normalized to $M / L_{B}=9.2$ (from van der Marel 1991; long dashed line) and $M / L_{B}=10.3$ (from Faber et al. 1997; short dashed line) respectively. The asterisks show the constant stellar $M / L_{B}=6$ assumed for luminous stars in all three galaxy models. Clearly, none of the model pairs exhibit a variation of $M / L_{B}\left(r_{e} / 3\right)$ as steep as the FP relation, but the dropout contribution to the FP slope is nevertheless consequential at this radius. However, the variation of $M / L_{B}$ at $r=480$ pc for the $q_{o}=1$ models follows the fundamental plane quite accurately over the range of $L_{B}$ between 4472 and $4472 / 4$. Note that the pair of variable dropout models with homologously scaled parameters $\left[\left(q_{o}, r_{d o}, m\right)=(4,2 \mathrm{kpc}, 1)\right.$ for 4472 and $\left(q_{o}, r_{d o}, m\right)=(4,0.667 \mathrm{kpc}, 1)$ for $\left.4472 / 4\right]$ produce $M / L_{B}(480)$ that vary in a sense opposite to the FP trend, but only when all cosmic gas from secondary infall is included. Our mass to light values for 4472 may be lower limits since we have not considered models in which unusually massive galaxies receive more than their cosmological allotment of halo material by tidal acquisitions from less massive, unmerged group galaxies.

The constant $q=q_{o}=1$ models can explain 
much of the FP tilt in $4472 \rightarrow 4472 / 4$ with or without secondary infall. However, when extended to $4472 / 16$, values of $M / L_{B}(480)$ clearly exceed the fundamental plane for all models. While the dropout contribution to $M / L_{B}(480)$ in the $q=1$ model (without cosmic inflow) decreases with $L_{B}$ over the range of 16 shown in Figure 2 (i.e. vertical distance between open squares and asterisks), the dropout mass does not disappear entirely for the 4472/16 model. In fact the assumed $M / L_{B}=6$ for the luminous stars alone (asterisks in Fig. 2) also lies above the FP for $4472 / 16$. Within the framework of our optically dark dropout assumption, this indicates that an independent variation of $M / L_{B}$ for the old stars would be required in addition to that provided by the dropout. Results shown in the upper panel of Figure 2 suggest that the FP could be fit over the full $4472 \rightarrow 4472 / 16$ range if we had chosen a somewhat lower value of $M / L_{B}$ for the luminous stars, but the X-ray data for NGC 4472 become difficult to fit if $M / L_{B} \lesssim 4$.

However, dynamically significant galactic rotation is expected for elliptical galaxies having Bband luminosities less than that of $4472 / 4,2 \times$ $10^{10} L_{B, \odot}$, which lies near the transition between massive, boxy elliptical galaxies with cores and low mass, rotationally supported, disky elliptical galaxies with power law profiles (Faber et al. 1997). Rotation can reduce the mass dropout in smaller galaxies since the cooled gas will be deposited in a relatively larger galactic volume, reducing $M / L$ values for $4472 / 16$ in Figure 2. Although the excess interstellar density in our models within $1-3 \mathrm{kpc}$ (Figure 1) can be understood in terms of $\sim 100 \mu \mathrm{G}$ magnetic fields, it could also indicate that we have somewhat overestimated the gas density in this region and also the mass of cooled gas.

Perhaps the best resolution of the large $M / L$ in our models of $4472 / 16$ would be to allow some of the cooled gas to form a luminous stellar population. Using this assumption, we have shown that the anomalously low ages for bright elliptical galaxies found from observations of the $\mathrm{H} \beta$ photometric index (e.g., Trager 1997) can be quite naturally produced by a young population with a Salpeter IMF extending up to $\sim 2 M_{\odot}$ but no further (Mathews \& Brighenti 1999a). In models having luminous dropout stars, about 10 percent of the optical light is produced by the young stars. The stellar disk component characteristic of rotating, low luminosity elliptical galaxies (Rix, Carollo, \& Freeman 1999), can be formed from younger cooling dropout stars (Brighenti \& Mathews 1997b), and there is some evidence that these galaxies also have enhanced $\mathrm{H} \beta$ indices (de Jong \& Davies 1997).

In our models we determine the detailed radial variation of the mass to light ratio within $r_{e}$; this variation depends most sensitively on our choice of $q(r)$. In general, optical observations of stellar velocity dispersion profiles cannot determine the detailed variation of $M / L$ with radius within $r_{e}$. Only with considerable effort have observers been able to detect the presence of dark matter at $r \gtrsim r_{e}$ (e.g., Carollo et al. 1995; Rix et al. 1997), although this dark matter is immediately apparent from X-ray observations (e.g. Brighenti \& Mathews 1997a). The insensitivity of optical observations to the detailed radial variation of $M / L$ is probably due to the generic stellar orbital bias toward radial anisotropy in $r \lesssim r_{e}$; this results in $M / L$ determinations that more nearly represent orbital averages rather than local values.

For completeness, in the final column of Table 1 we list the ROSAT X-ray luminosity of each of our models. In general our results are slightly flatter than the observed trend for luminous elliptical galaxies, $L_{x} \propto L_{B}^{p}$ with $p \approx 2$, in which the X-ray emission is interstellar (e.g. Eskridge, Fabbiano $\& \operatorname{Kim} 1995)$. However, we have shown elsewhere (Mathews \& Brighenti 1998) that $L_{x} / L_{B}$ correlates with the size of the X-ray image and that this correlation can be explained by the tidal transfer of halo material in galaxy groups from subordinate elliptical galaxies to the dominant central elliptical in the group. Galactic rotation can also influence $L_{x} / L_{B}$. Because of these environmental influences, the values of $L_{x}$ in Table 1 should not be regarded as a strong test of a particular model.

\section{FINAL REMARKS AND CONCLU- SIONS}

The full extent of the fundamental plane for elliptical galaxies spans a range of $L_{B}$ about $\sim 3$ times greater than the factor of 16 shown in Figure 2 (Burstein et al. 1997). Maintaining the FPHP tilt over this larger range would be difficult if cooling dropout is an important contributor of 
dark mass. Nevertheless, the presence of appreciable dropout mass seems unavoidable.

The total mass of cooled interstellar gas present in elliptical galaxies could be reduced by supernovadriven galactic winds or by the formation of stars that have since evolved. For our models we assume a Type Ia rate $\mathrm{SNu}(t)=\mathrm{SNu}\left(t_{n}\right)\left(t_{n} / t\right)^{p}$ supernovae per 100 years per $10^{10} L_{B}$ with $p=1$ and $\mathrm{SNu}\left(t_{n}\right)=0.03$ (Brighenti \& Mathews 2000a). Winds produced by more numerous Type Ia supernovae at early times (i.e. $p \gtrsim 1.3$ ) cannot be invoked to expel some of the dropout material from galaxies like $4472 / 4$ or $4472 / 16$ since these same higher Type Ia rates in more massive galaxies would greatly overproduce iron in the current interstellar gas, in violation of X-ray observations. Our value $\mathrm{SNu}\left(t_{n}\right)=0.03$ was chosen to be consistent with the low interstellar iron abundances for elliptical galaxies reported in most of the recent literature, $z_{F e, i s m} \lesssim 0.4$ solar (e.g., Tanaka et al. 1994). If the abundances are more nearly solar (Buote 1999), as is likely, then a proportionally larger $\mathrm{SNu}\left(t_{n}\right)$ is indicated; this can result in galactic winds in the $4472 / 16$ model at late times $t \sim t_{n}$. Therefore, the total mass of interstellar gas that cools can be reduced by SNIIdriven galactic outflow at early times (when most of the dropout occurs) and (less efficiently) by SNIa-driven outflow at late times. However, the large $L_{x}$ observed in virtually all bright elliptical galaxies clearly rules out ongoing galactic winds in these galaxies. The current amount of dropout mass could also be reduced if the IMF of the forming stars was more luminous in the past (i.e. more massive stars). While none of these means of reducing the dropout mass is securely understood, some significant mass of cooled gas must be present. The large mass of cooled interstellar gas expected within $r_{e}$ together with the alarming number of possible ways of enhancing or moderating the amount of cooled gas makes the coherency and tightness of the FP quite baffling.

Our main objective in presenting these results is to emphasize the importance of cooling dropout mass to the FP-HP controversy. The results that we have shown here are based on the commonly held assumption that only optically dark stars of very low mass can form in cooling flows. However, optically dark dropout by itself appears to be an unpromising explanation for the full FP tilt, although with some well chosen parameters that might be possible. What we see in Figure 2 is an unwelcome and quite unaesthetic disturbance of the $M / L$ relation, one that involves the uncertain physics of star formation and the origin of entropy fluctuations in the hot gas.

Nevertheless, the mass contribution from cooled interstellar gas cannot be dismissed by those concerned with the deviation of the fundamental plane from a truly homologous plane. Like other effects proposed to explain the FP-HP discrepancy, if the cooling dropout mass is a dominant contributor, the mass dropout must vary in a smooth, regular fashion across the FP, proportional to some power of $L_{B}$. Such regularity could conceivably result from the similar profiles of interstellar gas density and temperature observed in the central regions of elliptical galaxies $\left(r \lesssim r_{e}\right)$, provided the entropy fluctuations are also similar. Our apparent success in explaining the the anomalously young ages of elliptical galaxies indicated by the $\mathrm{H} \beta$ index from an optically luminous dropout stellar population (Mathews \& Brighenti 1999b) strongly suggests that a better match to the FP-HP tilt could be achieved if the optically dark assumption used here is relaxed. In this case the IMF of the continuously forming stars is likely to extend to higher maximum masses in elliptical galaxies of lower $L_{B}$ because of the lower interstellar pressure in low luminosity elliptical galaxies.

Studies of the evolution of hot gas in elliptical galaxies at UC Santa Cruz is supported by grants from NASA and the NSF for which we are very grateful. In addition FB is supported in part by Grant MURST-Cofin 98. 


\section{REFERENCES}

Brighenti, F. \& Mathews, W. G. 1997a, ApJ 486, L83

Brighenti, F. \& Mathews, W. G. 1997b, ApJ 490, 592

Brighenti, F. \& Mathews, W. G. 1999a, ApJ 512, 65

Brighenti, F. \& Mathews, W. G. 2000a, ApJ (in press) (astro-ph/0002385)

Brighenti, F. \& Mathews, W. G. 2000b, ApJ (submitted)

Buote, D. A. 1999, MNRAS 309, 685

Burkert, A. \& Silk, J. 1997, ApJ 488, L55

Burstein, D., Bender, R., Faber, S., \& Nolthenius, R. 1997, AJ, 114, 1365

Busarello, G., Capaccioli, M., Capozziello, S., Longo, G. \& Puddu, E. 1997, A\&A 320, 415

Capelato, H. V., de Carvalho, R. R., \& Carlberg, R. G. 1995, ApJ 451, 525

Carollo, C. M., de Zeeuw, P. T., van der Marel, R. P., Danziger, I. J. \& Qian, E. E. 1995, ApJ 441, L25

Ciotti, L. 1991, A\&A 249, 99

Ciotti, L. \& Lanzoni, B. 1997, A\&A 321, 724

Ciotti, L., Lanzoni, B. \& Renzini, A. 1996, MNRAS 282,1

de Jong, R. S. \& Davies, R. L. 1997, MNRAS 285, L1

Djorgovski, M. 1995, ApJ 438, L29

Djorgovski, M. \& Davis, S. 1987, ApJ 313, 59

Dressler, A., Lynden-Bell, D., Burstein, D., Davies, R. L., Faber, S. M., Terlevich, R. J. \& Wegner, G. 1987, ApJ 313, 42

Ekers, R. \& Kolanyi, C. A\&A 67, 47

Eskridge, P. B., Fabbiano, G. \& Kim, D.-W. 1995, ApJS 97, 141

Faber, S. M., Tremaine, S., Ajhar, E. A., Byun, Y-I., Dressler, A., Gebhardt, K., Grillmair, C., Kormendy, J., Lauer, T. R., \& Richstone, D. 1997, AJ 114, 1771

Fabian, A. C., Nulsen, P. E. J. \& Canizares, C. R. 1982, MNRAS 201, 933
Ferland, G. J., Fabian, A. C. \& Johnstone, R. M. 1994, MNRAS 266, 399

Graham, A. W. 1998, MNRAS 295, 933

Graham, A. W. \& Colless, M. 1997, MNRAS 287, 221

Hanlan, P. C. \& Bregman, J. N. 2000, ApJ (in press) (astro-ph/9910210)

Hjorth, J. \& Madsen, J. 1995, ApJ, 445, 55

Irwin, J. A. \& Sarazin, C. L. 1996, ApJ 471, 683

Kravtsov, A. V., Klypin A. A., Bullock, J. S. \& Primack, J. R. 1998, ApJ 502, 48

Macchetto, F. et al. 1996, A\&AS 120, 463

Magorrian, J. et al. 1998, ApJ 115, 2285

Mathews, W. G. \& Brighenti, F. 1998a, ApJ 493, L9

Mathews, W. G. \& Brighenti, F. 1998b, ApJ 503, L15

Mathews, W. G. \& Brighenti, F. 1999a, ApJ 526, 114

Mathews, W. G. \& Brighenti, F. 1999b, ApJ 527, L31

Navarro, J. F., Frenk, C. S. \& White, S. D. M. 1996, ApJ, 462, 563

Navarro, J. F. \& Steinmetz, M. 2000, ApJ 528, 607

Pahre, M. A., de Carvalho, R. R. \& Djorgovski, S. G. 1998, AJ 116, 1606

Pahre, M. A., Djorgovski, S. G. \& de Carvalho, R. R. 1995, ApJ 453, L17

Pahre, M. A. \& Djorgovski, S. G. 1997, in The Nature of Elliptical Galaxies, Proceedings of the Second Stromlo Symposium, eds. M. Arnaboldi, G. S. Da Costa \& P. Saha, ASP Conf. Ser. Vol. 116, (San Francisco:ASP), 154

Prugniel, P. \& Simien, F. 1997, A\&A 321, 111

Renzini, A. \& Ciotti, L. 1993, ApJ 416, L49

Rix, H.-W., de Zeeuw, P. T., Cretton, N., van der Marel, R. P. \& Carollo, C. M. 1997, ApJ, 488, 702

Rix, H.-W., Carollo, M. \& Freeman, K. 1999, ApJ 513, L25

Sarazin, C. L. \& Ashe, G. A. 1989, ApJ 345, 22 
Scodeggio, M., Gavazzi, G., Belsole, E., Pierini, D., \& Boselli, A. 1998, MNRAS 301, 1001

Tanaka, Y., Inoue, H. \& Holt, S. S. 1994, PASJ 46, L37

Trager, S. 1997, PhD thesis, University of California Santa Cruz

Trinchieri, G., Fabbiano, G. \& Canizares, C. R. 1986, ApJ 310, 637

van der Marel, R. P. 1991, MNRAS, 253, 710

Worthey, G. 1994, ApJS 95, 107

Worthey, G., Trager, S., \& Faber, S. M. 1996, in ASP Conf. Ser. 86, Fresh Views of Elliptical Galaxies, eds. A. Buzzoni \& A. Renzini (San Francisco:ASP), 203

This 2-column preprint was prepared with the AAS LATEX macros v5.0. 
TABLE 1

COOLING DROPOUT PARAMETERS AND MASS TO LIGHT RATIOS

\begin{tabular}{|c|c|c|c|c|c|c|c|c|}
\hline GALAXY & $\begin{array}{c}L_{B} \\
\left(10^{10} L_{B, \odot}\right)\end{array}$ & $q_{o}$ & $\begin{array}{r}r_{d o}{ }^{\mathrm{a}} \\
(\mathrm{kpc})\end{array}$ & $m^{\mathrm{a}}$ & $\begin{array}{c}\text { COSMIC }^{\mathrm{b}} \\
\text { GAS? }\end{array}$ & $\frac{M}{L_{B}}\left(\frac{r_{e}}{3}\right)$ & $\frac{M}{L_{B}}(480 \mathrm{pc})$ & $\begin{array}{c}L_{x}^{\mathrm{c}} \\
\left(10^{40} \mathrm{ergs} \mathrm{s}^{-1}\right)\end{array}$ \\
\hline \multicolumn{9}{|l|}{ 4472/16: } \\
\hline & 0.493 & 0 & $\cdots$ & $\cdots$ & $\mathrm{NO}$ & 9.32 & 8.57 & 0.10 \\
\hline & 0.493 & 1 & $\infty$ & $\cdots$ & NO & 7.29 & 7.13 & 0.10 \\
\hline & 0.493 & 4 & 0.317 & 1 & $\mathrm{NO}$ & 8.25 & 8.17 & 0.25 \\
\hline \multicolumn{9}{|l|}{ 4472/4: } \\
\hline & 1.97 & 0 & $\cdots$ & $\cdots$ & YES & 11.76 & 19.27 & 9.35 \\
\hline & 1.97 & 0 & $\cdots$ & $\cdots$ & $\mathrm{NO}$ & 8.97 & 12.37 & 1.56 \\
\hline & 1.97 & 1 & $\infty$ & $\cdots$ & YES & 7.95 & 8.41 & 8.00 \\
\hline & 1.97 & 1 & $\infty$ & $\cdots$ & $\mathrm{NO}$ & 7.40 & 7.65 & 1.52 \\
\hline & 1.97 & 4 & 0.667 & 1 & YES & 11.05 & 10.91 & 14.57 \\
\hline & 1.97 & 4 & 0.667 & 1 & $\mathrm{NO}$ & 8.65 & 8.54 & 2.57 \\
\hline & 1.97 & 4 & 2 & 1 & YES & 8.16 & 7.27 & 10.78 \\
\hline & 1.97 & 4 & $\infty$ & $\cdots$ & YES & 7.03 & 6.79 & 6.00 \\
\hline 44r2: & 7.89 & 0 & $\cdots$ & $\ldots$ & YES & 10.47 & 32.29 & 20.08 \\
\hline & 7.89 & 0 & $\ldots$ & $\ldots$ & $\mathrm{NO}$ & 9.97 & 28.53 & 8.59 \\
\hline & 7.89 & 1 & $\infty$ & $\ldots$ & YES & 8.83 & 11.40 & 31.56 \\
\hline & 7.89 & 1 & $\infty$ & $\ldots$ & $\mathrm{NO}$ & 8.64 & 10.96 & 12.56 \\
\hline & 7.89 & 4 & 2 & 1 & YES & 9.74 & 7.99 & 28.11 \\
\hline & 7.89 & 4 & 2 & 1 & $\mathrm{NO}$ & 9.37 & 7.88 & 13.73 \\
\hline & 7.89 & 4 & 0.8 & 2 & YES & 10.47 & 13.08 & 36.44 \\
\hline & 7.89 & 4 & $\infty$ & $\cdots$ & YES & 7.88 & 7.20 & 39.82 \\
\hline
\end{tabular}

${ }^{\text {a When }} q(r)=q_{o}$ is constant, the exponential factor in equation (3) is set to unity, as if $r_{d o}=\infty$ and $m$ is undefined.

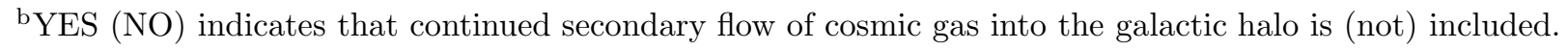

${ }^{\mathrm{c}}$ ROSAT band X-ray luminosity (including dropout emission) within $150 \mathrm{kpc}$ for NGC 4472, $50 \mathrm{kpc}$ for $4472 / 4$ and $16.6 \mathrm{kpc}$ for $4472 / 16$ 
Fig. 1.- Top panel: Comparison of radial interstellar density variation for two hydrodynamic models at time $t_{n}=13$ Gyrs with observed density in NGC 4472. Filled circles are Einstein data (Trinchieri, Fabbiano, \& Canizares 1986), open circles are from ROSAT (Irwin \& Sarazin 1996). The light solid and dashed lines refer to the background (uncooled) gas density in the $q_{o}=1$ and $\left(q_{o}, r_{d o}, m\right)=(4,2 \mathrm{kpc}, 1)$ models respectively. The heavy lines are the apparent space variation of the gas density including emission from gas that is cooling locally. Bottom panel: Comparison of the interstellar temperature variation with radius computed in the same two models with ROSAT temperature data (Irwin \& Sarazin 1996). The light solid and dashed lines refer to the computed temperature variation in the uncooled background flow with physical radius. The heavy solid and dashed lines show the apparent variation of temperature projected on the sky and corrected for emission from mass dropout; these curves should be compared with the observations.

Fig. 2.- Variation of the mass to light ratio $M / L_{B}$ with luminosity $L_{B}$ (both in solar units) at $t_{n}=13$ Gyrs. $M / L_{B}$ includes both luminous stars and interstellar dropout for NGC 4472 (on the right) and two homologously scaled galaxies having $1 / 4$ and $1 / 16$ the mass of NGC 4472 (center and left respectively). Top panel: $M / L_{B}$ evaluated within fixed radius $r=480 \mathrm{pc}$ for all galaxies; Bottom panel: $M / L_{B}$ within $r=r_{e} / 3$ for all galaxies. Asterisks show $M / L_{B}=6$ assumed for the luminous stars. Dashed lines show the local the fundamental plane, $M / L_{B} \propto L_{B}{ }^{0.24}$, normalized at NGC 4472 with $M / L_{B}=9.2$ (long dashed line) or $M / L_{B}=10.3$ (short dashed line). Circles refer to no-dropout models $\left(q_{o}=0\right)$, squares to uniform $q_{o}=1$ models, triangles to uniform $q_{o}=4$ models and hexagons to variable dropout models with $\left(q_{o}, r_{d o}, m\right)=(4,2 \mathrm{kpc}, 1)$ for 4472 and $\left(q_{o}, r_{d o}, m\right)=(4,0.667 \mathrm{kpc}, 1)$ for $4472 / 4$ (upper filled hexagon) or $\left(q_{o}, r_{d o}, m\right)=(4,2 \mathrm{kpc}, 1)$ for 4472/4 (lower filled hexagon). Gas from secondary cosmic infall is included in solutions with filled symbols and this gas is excluded in solutions with open symbols. Where possible the symbols are labeled with $\left(q_{o}, r_{d o}, m, Y / N\right) ; Y / N$ refers to the presence or lack of secondary infall. 

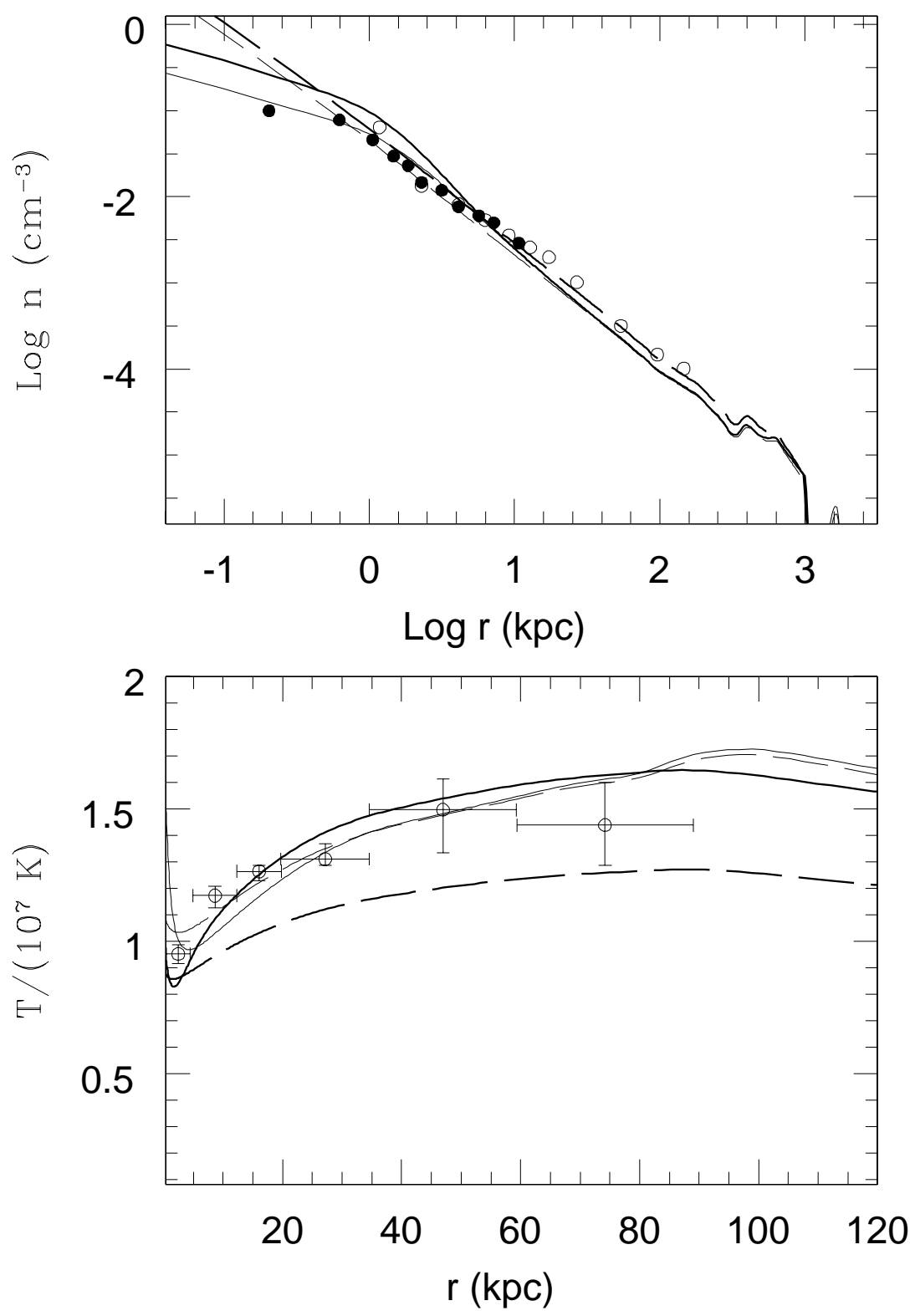


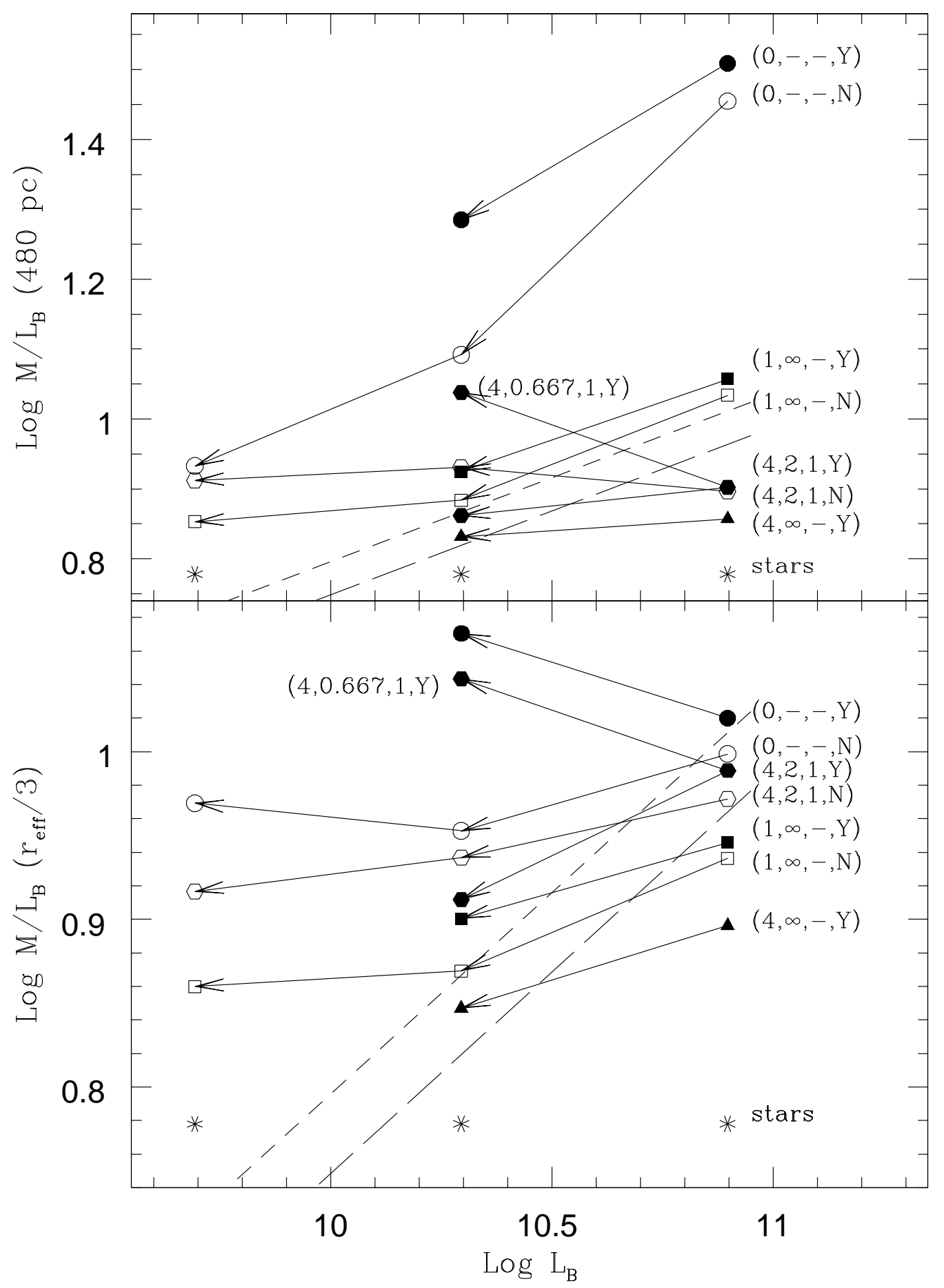

